\title{
Transition length between water and air-water flows on stepped chutes
}

\author{
A. L. A. Simões ${ }^{2}$, H. E. Schulz ${ }^{1,2}$ \& R. M. Porto ${ }^{2}$ \\ ${ }^{1}$ Nucleus of Thermal Engineering and Fluids, \\ School of Engineering at São Carlos, University of São Paulo, Brazil \\ ${ }^{2}$ Department of Hydraulics and Sanitary Engineering, \\ School of Engineering at São Carlos, University of São Paulo, Brazil
}

\begin{abstract}
This study presents the steps followed to obtain mathematical models for the length of the transition region between the "full-water" and "full-mixed" flows in stepped spillways. This transition length is defined here as the distance along the flow, parallel to the pseudo-bottom, starting at the end of the "full-water" region and ending at the beginning of the "full-mixed" region. The definition is proposed based on experimental profiles of the surface obtained with an acoustic sensor in a stepped chute, which allows one to locate adequately the minima and maxima of the profile. A set of profiles obtained for different flow conditions is shown, and a comparison between predicted and calculated transition lengths is made. Experimental data and theoretical predictions superpose adequately for the present set of data.
\end{abstract}

Keywords: air-water flow, aeration, transition length, stepped spillways.

\section{Introduction}

For the design of stepped chutes it is necessary to know the behaviour of the airwater mixture along the flow. In many cases it is necessary to build bottom aerators that must be conveniently placed, for which the position of the inception point is needed (the section where the air begins to be captured by the water at the upper surface). It is also necessary to know the length of the black water region (upflow of the inception point), and the length along the flow needed for the air to travel until the bottom of the channel (downflow of the inception point). This length is here called "transition length", and is defined as the 
distance, parallel to the pseudo-bottom, which starts at the end of black water region and finishes at the beginning of the full-aerated region. The present analysis is valid for skimming flows.

It is generally accepted that skimming flows, as occur in smooth chutes or spillways, consists of three regions: (1) single-phase flow (water); (2) partially aerated flow, and (3) air-water two phase flow (Cain and Wood [5]). Similarly, these regions are also observed in stepped chutes, and their quantification is relevant, being motivated by the need of protection of the spillways from possible damages caused by cavitation. This study presents a mathematical model for the transition length in stepped spillways.

\section{Theoretical formulation}

Two formulations are presented to obtain predictions of the Transition length. The first one considers classical conservation principles, and the second considers an approximation based on analogies with basic transport equations.

\subsection{Equation based on conservation principles (Model 1)}

In this approximation, the principles of conservation were written in the Eulerian and integral forms. The control volume chosen has two input surfaces (section 1 and the free surface, through which air is captured), and one output surface (section 2), as shown in fig. 1. It is assumed that the main transport of mass, momentum and energy occurs through sections 1 and 2, as shown in the sequence. For steady state calculations, the integrations are performed over the areas of sections 1 and 2 .

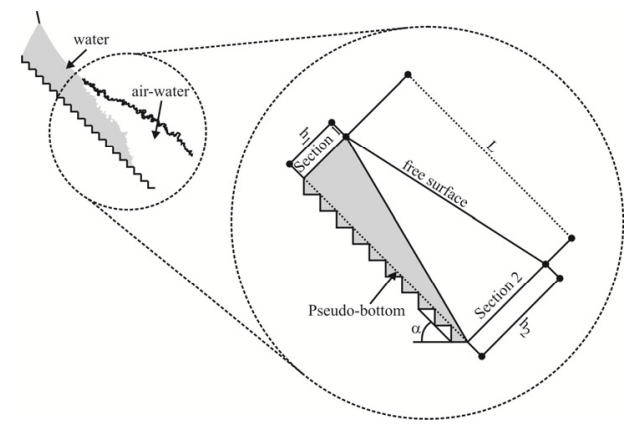

Figure 1: Control volume.

\subsubsection{Conservation of mass}

The mass conservation equation for steady flow is:

$$
\iint_{1} \rho_{1} v_{1} d A+m_{\text {Surf }}=\iint_{2} \rho_{2} v_{2} d A
$$

$\rho, v$, and $A$ represent the densities, velocities and areas considered in the problem. The subscripts "Surf", 1 and 2 indicate the upper surface, and sections 
1 and 2, respectively, while $m$ represents the air mass flux. Because this flux is much lower than those given by the integrals on surfaces 1 and 2, its influence is considered here as composing the corrective factor of the flux on section 2 .

As $\rho_{1}$, is constant, but $\rho_{2}$ varies along $A_{2}$, we have:

$$
\rho_{1} \iint_{1} v_{1} d A=\rho_{1} V_{1} A_{1} \quad \text { and } \quad \frac{\iint_{2} \rho_{2} v_{2} d A}{A_{2}} \neq \bar{\rho}_{2} V_{2}
$$

The use of average values imposes a corrective factor $\left(\omega_{0}\right)$, which also accounts for $m_{\text {Surf }}$ of eqn (1), furnishing

$$
\omega_{0} \bar{\rho}_{2} V_{2} A_{2}=\rho_{1} V_{1} A_{1}
$$

Defining $C$ as the volume fraction occupied by the air, the density of the mixture $\left(\rho_{2}\right)$ is usually presented as:

$$
\bar{\rho}_{2}=\bar{C} \rho_{g}+(1-\bar{C}) \rho_{1} \Rightarrow \frac{\bar{\rho}_{2}}{\rho_{1}} \cong(1-\bar{C}) \text { for } \frac{\bar{C} \rho_{g}}{\rho_{1}}<(1-\bar{C})
$$

Substituting 4 into 3, and using $A=B h$, leads to:

$$
\frac{V_{2}}{V_{1}}=\frac{1}{\omega_{0}(1-\bar{C}) h^{*}}
$$

where $h^{*}=h_{2} / h_{1}$.

\subsubsection{Conservation of momentum}

The momentum equation for steady flow is given by:

$$
F=-\iint_{1} \rho_{1} v_{1} v_{1} d A+\iint_{2} \rho_{2} v_{2} v_{2} d A=-\beta_{1} \rho_{1} V_{1}^{2} B h_{1}+\omega_{1} \bar{\rho}_{2} V_{2}^{2} B h_{2}
$$

$\beta_{1}$ is the Boussinesq coefficient, and $\omega_{1}$ in section 2 is a weighting factor which takes into account the fact that $\rho \neq \bar{\rho}_{2}$. The control volume has a lateral trapezoidal form, as shown in fig. $1 . \omega_{2}$ is defined as the fraction of this area occupied by water (ideally corresponding to the lower triangle in the figure). It is assumed that the mixture at the remaining fraction of the trapezoid (ideally the upper triangle) is $\bar{\rho}_{2}$, defined by eqn (5). The force $F$ acting on the control volume is expressed as $F=F_{w}+F_{p}+F_{o}$ where $F_{w}$ is the weight, $F_{p}$ is the force due to pressure and $F_{\tau}$ is the force due to the bottom shear stress. The three forces are given, respectively, by:

$$
F_{w}=\frac{\left(h_{1}+h_{2}\right)}{2} B L \rho_{1} g\left[\omega_{2}+\left(1-\omega_{2}\right)(1-\bar{C})\right] \sin \alpha
$$




$$
\begin{gathered}
F_{p 1}-F_{p 2}=\frac{\rho_{1} g}{2} B\left[h_{1}^{2}-(1-\bar{C}) h_{2}^{2}\right] \cos \alpha \\
F_{\tau}=-\tau_{o} B L
\end{gathered}
$$

The effects of shear stresses on the walls and the free surface were not considered. Combining eqns (5), (6) and (7), solving them for $L / h_{1}$, defining the nondimensional parameters $L^{*}=L / h_{1}$, and $F r_{1}=V /\left(g h_{1}\right)^{1 / 2}$, leads to:

$$
L^{*}=\frac{-\beta_{1}+\frac{\omega_{1}}{\omega_{0}^{2}} \frac{1}{(1-\bar{C}) h^{*}}-\frac{1}{2 F r_{1}^{2}}\left[1-(1-\bar{C}) h^{* 2}\right] \cos \alpha}{\frac{\left(1+h^{*}\right)}{2 F r_{1}^{2}}\left[\omega_{2}+\left(1-\omega_{2}\right)(1-\bar{C})\right] \sin \alpha-\frac{f}{8}}
$$

The shear stress was obtained from the Darcy-Weisbach equation, and is related to the friction factor $f$ at the bottom of the channel as:

$$
f=\frac{\tau_{o}}{\frac{1}{8} \rho_{1} V_{1}^{2}}
$$

\subsubsection{Conservation of energy}

The energy equation for steady flow is given by:

$$
\dot{Q}-\dot{W}=-\iint_{1} \rho_{1}\left(e_{1}+\frac{p_{1}}{\rho_{1}}\right) v_{1} d A-\dot{E}_{\text {Surf }}+\iint_{2} \rho_{2}\left(e_{2}+\frac{p_{2}}{\rho_{2}}\right) v_{2} d A
$$

$\dot{Q}$ is the heat transfer across the control surfaces, $\dot{W}$ is the power transferred due to shear forces, $\dot{E}_{\text {Surf }}$ is the contribution due to the air flow through the surface (neglected here in relation to the other parcels), and $e=g z+v^{2} / 2+u$. Eqn (10) is then represented as:

$$
\begin{aligned}
\dot{Q}-\dot{W}=-\rho_{1} \int & \iint_{1}\left(g z_{1} v_{1}+\frac{v_{1}^{3}}{2}+u_{1} v_{1}+\frac{p_{1} v_{1}}{\rho_{1}}\right) d A+ \\
& +\iiint_{2}\left(g \rho_{2} z_{2} v_{2}+\rho_{2} \frac{v_{2}^{3}}{2}+\rho_{2} u_{2} v_{2}+p_{2} v_{2}\right) d A
\end{aligned}
$$

The integrals at sections 1 and 2 are represented, in the next equations, by $I_{1}$ and $I_{2}$, respectively. Using mean values, a Coriolis coefficient $\alpha_{c 1}$ must be used for section 1 , and $p_{1}=\rho_{1} g h$. In section 2 a new weight coefficient $\omega_{3}$ is used (similar to the Coriolis coefficient), $z_{2}=0$ and $p_{2}$ is assumed to be $p_{2}=\omega_{4} \bar{\rho}_{2}, g h$, where $\bar{\rho}_{2}$ is the mean value of $\rho_{2}$ and $\omega_{4}$ is an adjusted constant. 


$$
\begin{aligned}
& I_{1}=g\left(L \sin \alpha+h_{1} \cos \alpha\right) V_{1} A_{1}+\alpha_{c 1} \frac{V_{1}^{3}}{2} A_{1}+\bar{u}_{1} V_{1} A_{1} \\
& I_{2}=\frac{\omega_{4} \cos \alpha}{2} g \bar{\rho}_{2} h_{2} V_{2} A_{2}+\omega_{3} \bar{\rho}_{2} \frac{V_{2}^{3}}{2} A_{2}+\bar{\rho}_{2} \bar{u}_{2} V_{2} A_{2}
\end{aligned}
$$

Combining eqns (3), (5), (10) and (11):

$$
h_{p}=\omega_{0}\left[\left(\operatorname{Lsen} \alpha+h_{1} \cos \alpha\right)+\alpha_{c 1} \frac{V_{1}^{2}}{2 g}\right]-\left(\omega_{3} \frac{V_{2}^{2}}{2 g}+\omega_{4} \cos \alpha \frac{h_{2}}{2}\right)
$$

Where $h_{p}$, known as "head loss", is given by:

$$
h_{p}=-\frac{\dot{Q}-\dot{W}_{r}+\rho_{1} \bar{u}_{1} V_{1} A_{1}-\bar{\rho}_{2} \bar{u}_{2} V_{2} A_{2}}{g \bar{\rho}_{2} V_{2} A_{2}}
$$

The head loss may be simplified to a punctual loss, so that $h_{p}=K F_{r 1}{ }^{2} h_{1} / 2$, where $K$ is a constant. Writing then eqn (12) in nondimensional form, and solving it for $1-\bar{C}$, results:

$$
(1-\bar{C})=\frac{\sqrt{\omega_{3}}}{\omega_{0}}\left[h^{*} \sqrt[\begin{array}{l}
\omega_{0} \frac{2 L^{*} \operatorname{sen} \alpha}{F r_{1}^{2}}+ \\
+\frac{2}{F r_{1}^{2}}\left(\omega_{0}-\omega_{4} \frac{h^{*}}{2}\right) \cos \alpha+\omega_{0} \alpha_{c 1}-K
\end{array}]{]^{-1}}\right.
$$

The set of eqns (8) and (14) allows to obtain $L^{*}$, and is here named "Model 1 ". In this study a least squares adjustment of the constants was made using a nonlinear procedure.

\subsection{Analogy with basic transport equations (Model 2)}

Schulz and Simões [3] presented an alternative analysis of the transition region also considering the volume of fig. 1 . The authors considered that: 1 ) the rate of formation of voids in the water, indicated by $\dot{c}$, is proportional to the water flow rate that crosses the volume, $q$; and 2) $\dot{c}$ is also proportional to the slope of the surface, $d h / d x$. Joining both proportionalities, it leaded to:

$$
\dot{c}=K q \frac{d h}{d x}
$$

$K$ is a proportionality factor. The dimensions of the variables are: $|h|=\mathrm{m},|x|=\mathrm{m}$, $|q|=\mathrm{m}^{2} \mathrm{~s}^{-1},|\dot{c}|=\mathrm{s}^{-1},|K|=\mathrm{m}^{-2}$. As mentioned by Schulz and Simões [3], eqn (15) is similar to the basic equations used in Transport Phenomena for mass and heat 
transfer, because it involves a first order derivative. Assuming $\dot{c}, q$ and $K$ as constants, the integration of this equation, from $x=0$ to $x=L$, produces:

$$
L=\frac{K q}{\dot{c}}\left(h_{2}-h_{1}\right)
$$

Although very simple, eqn (16) produced a correlation coefficient of about 0.73 (considered acceptable) when compared with the experimental data described in section 3. It was then "allowed" for one of the constants to vary. $K$ was chosen, and the following general form was proposed:

$$
K=\sum_{i} \phi_{i}(q h)^{i}
$$

$\phi_{i}$ are constants. Eqns (15) and (17) were then rearranged to:

$$
\dot{c}=\sum_{i} \phi_{i}(q h)^{i} \frac{d(q h)}{d x}
$$

To obtain an adequate solution, it is necessary to conveniently truncate the series in eqn (18). For $i=0$, the solution is eqn (16) (acceptable). In this study we used $i=1$, which leads to:

$$
L=\frac{\phi_{0}}{\dot{c}}\left(q h_{2}-q h_{1}\right)+\frac{\phi_{1}}{\dot{c}}\left[\left(q h_{2}\right)^{2}-\left(q h_{1}\right)^{2}\right]
$$

This equation was compared with the measured data, together with an alternative semi-empirical form, in which all parcels have independent coefficients, that is,

$$
L=\theta_{1} q h_{2}+\theta_{2} q h_{1}+\theta_{3}\left(q h_{2}\right)^{2}+\theta_{4}\left(q h_{1}\right)^{2}+\theta_{5}
$$

$\theta_{i}$ are constants. Eqn (20) has the same form of eqn (19), which is the reason of using it here. It is obviously expected that the last equation furnishes a better fit to experimental data, because it involves five adjustment constants (the former involves only two). Eqn (19) is named here Model 2a and eqn (20) is named Model 2b.

\section{Experimental results}

Experiments were conducted in a channel of the Laboratory of Environmental Hydraulics, in the School of Engineering at São Carlos (fig. 2a). The channel was $5 \mathrm{~m}$ long and $20 \mathrm{~cm}$ wide, allowing adjustments of the slope angle between $0^{\circ}$ and $45^{\circ}$. In this study $\alpha=45^{\circ}$. The height of the steps was $s=5 \mathrm{~cm}$. The flow rate was controlled upstream by a sluice gate, and measured with an electromagnetic flowmeter. The profiles of the free surface were obtained positioning an ultrasonic sensor in 40 locations along $3.5 \mathrm{~m}$ of the channel. The sampling frequency adopted for the depth measurements was $50 \mathrm{~Hz}$, and the sensor was maintained $120 \mathrm{~s}$ at each position to obtain representative data for the 
depths. A typical mean profile obtained in this study is shown in fig. 2b. This figure also shows the plot of the analytical solution for the single phase region (indicated by $\mathrm{S}_{2}$, as obtained by Simões et al. [4]), and the minimum and maximum positions that limit the transition region.
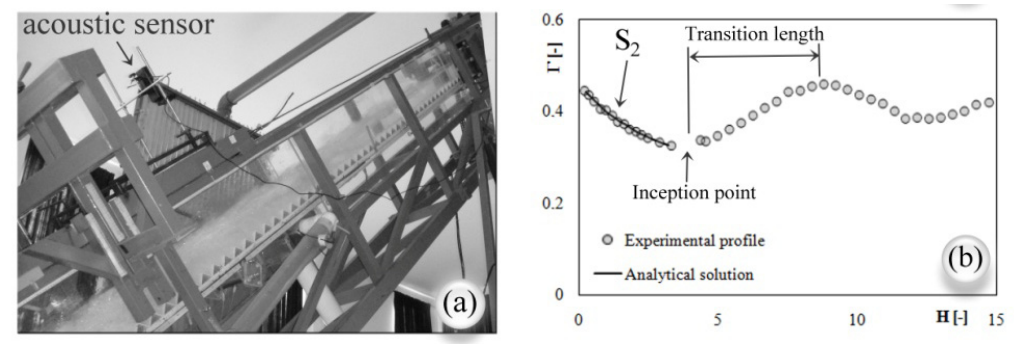

Figure 2: (a) Stepped chute and (b) experimental profile. The transition length is the distance between the minimum and maximum points of the surface profile.

\subsection{Comparison with literature}

The position of the inception point and the length of the transition region were here obtained from the measurements, as indicated in fig. 2b. A comparison was made with a prediction for the position of the inception point suggested by Boes and Hager [2]. Following a translation of the origin similar to that proposed by Boes [1], and using the measured points to adjust the theoretical curve, the difference between the positions of the measured and predicted inception points corresponds roughly to the transition length. The difference occurs because Boes and Hager [2] considered the full mixed region, while here the beginning of the transition region is considered.

\subsection{Measured and predicted transition lengths}

Figs 3, 4 and 5 show all the surface profiles measured in the present study. It is shown that the minima and maxima of the different profiles are well defined in most of the experiments, which points to the adequacy of this methodology. In some profiles the measurements are somewhat sparser, like shown in figs $5 \mathrm{n}$ (minimum) and 5o (maximum), which introduce errors in the evaluation of $L, h_{1}$ and $h_{2}$, but even so allowing to observe the transition region.

The proposed models for the transition length involve sets of constants that must be adjusted. For eqns (8) and (14) (Model 1), a nonlinear least squares adjustment using the set of experimental data furnished: $\alpha_{c 1}=3.61 ; \beta_{1}=1.44 ; \omega_{3}$ $=0.042 ; \omega_{1}=0.086 ; K=0.17 ; \omega_{2}=1 ; f=0.0 ; \omega_{0}=0.056 ; \omega_{4}=0.91$. It must be said that other constants may also produce good adjustments, and that the physical principles used to derive the equations imposed the definition of this set of constants. The superposition between model and experimental data is shown 

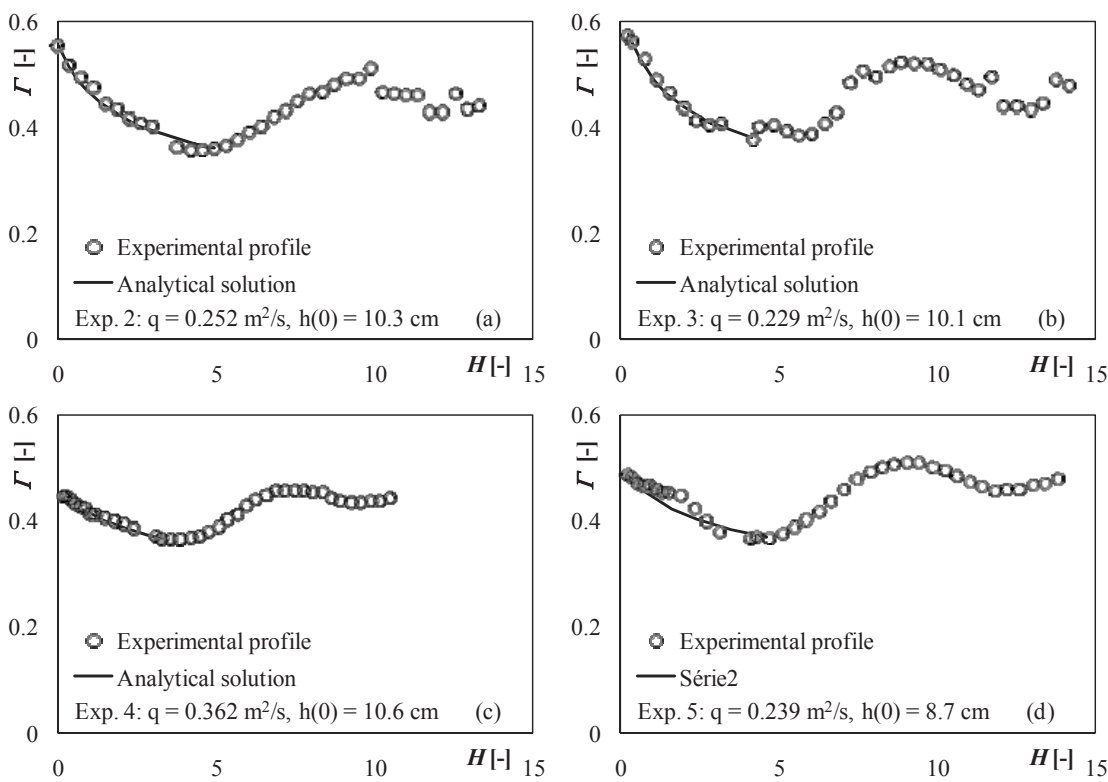

Figure 3: $\quad$ Experimental profiles and analytical solutions for runs 2 through 5. in fig. 6a. As can be seen, the general trend of the experiments was captured by the model.

The convenience of a more restricted set of constants was one of the reasons for the search of an alternative quantification (Schulz and Simões [3]). Applying the least squares method to eqn (19) (Model 2a), the following constants were obtained: $\phi_{0} / \dot{c}=290.7, \phi_{1} / \dot{c}=-2290.1$. As can be seen in fig. $6 \mathrm{~b}$, also this equation follows the general trend of the experimental data.

Finally, applying the least squares method to eqn 20 (Model 2b), the following constants were obtained: $\theta_{1}=789.12, \theta_{2}=-976.5, \theta_{3}=-10610.5, \theta_{4}=$ 17265.15, $\theta_{5}=0.1923$. Considering the number of constants, Model $2 \mathrm{~b}$ (five constants) lies between Model 1 (nine constants) and Model 2a (two constants). Fig. 6c shows that it reproduces well the experimental data, and that it may be considered in further studies for the quantification of transitions lengths.

The correlation coefficients between measured and predicted values were: Model 1 $=0.94$, Model 2a $=0.90$, and Model $2 \mathrm{~b}=0.97$.

\section{Conclusions}

Governing equations were derived for the calculation of transition lengths between full-water and full-mixed regions in stepped spillways. Two main procedures were followed: 1) using conservation principles, and 2) using an analogy with basic transport equations. The second procedure furnished an equation with two adjustment constants, which was modified, involving then five constants. The three predictions were compared with experimental data obtained 

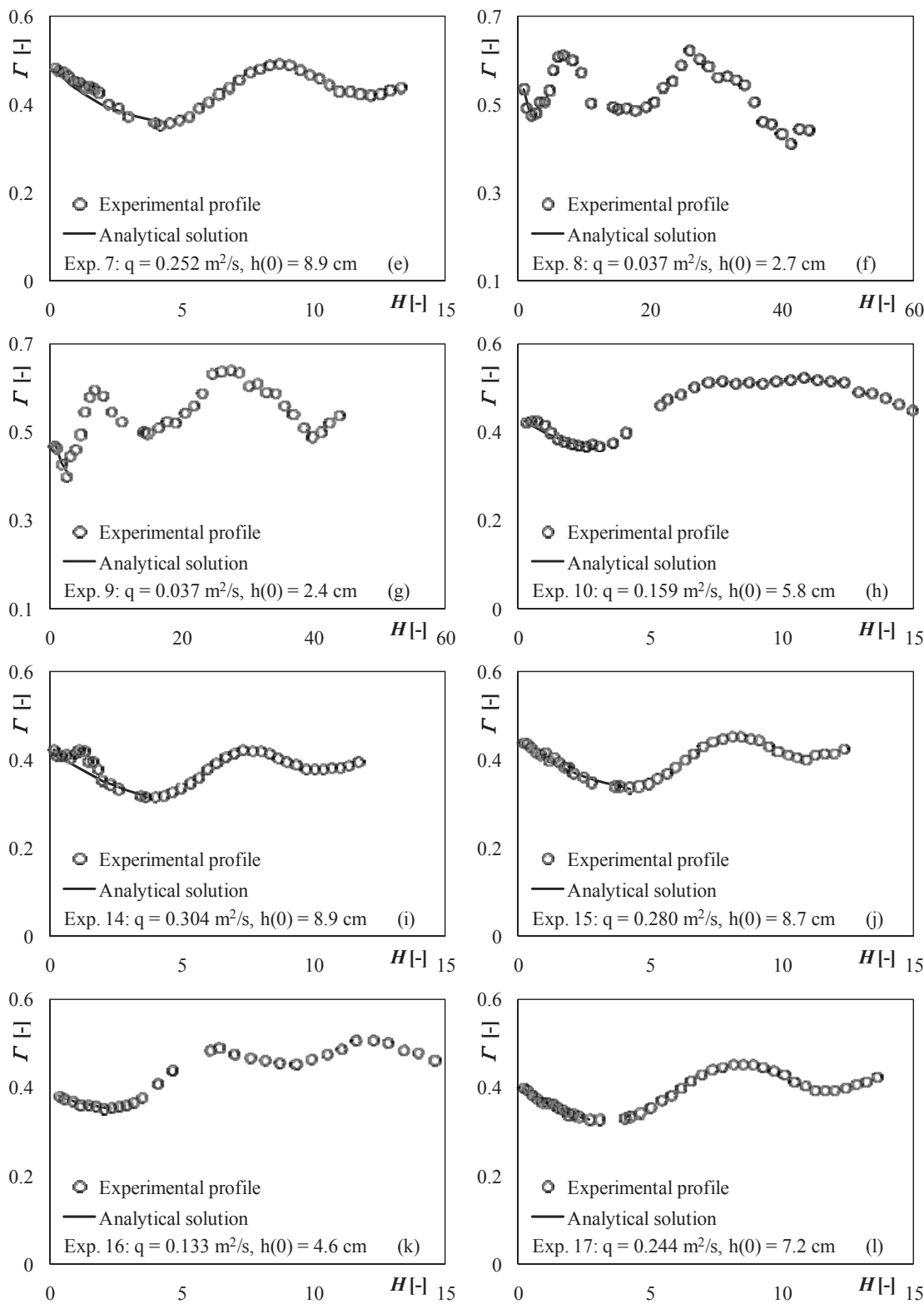

Figure 4: Experimental profiles and analytical solutions for runs 7 through 10, and 14 through 17. 

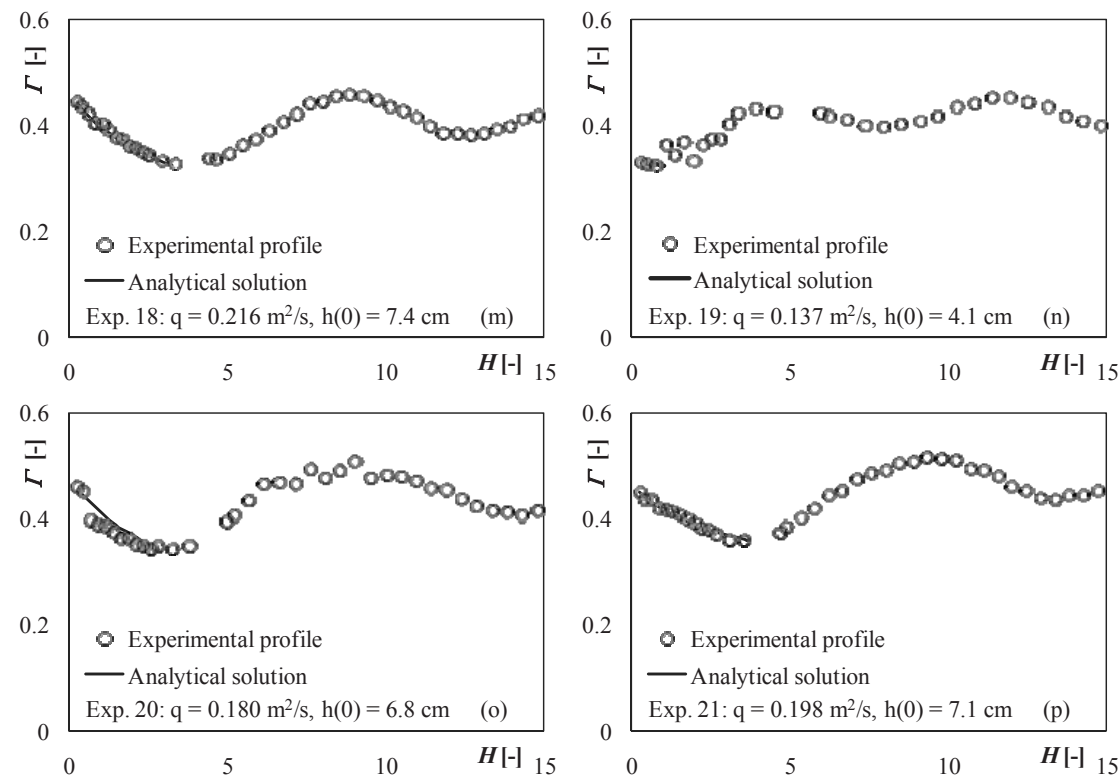

Figure 5: Experimental profiles and analytical solutions for runs 18 through 21.
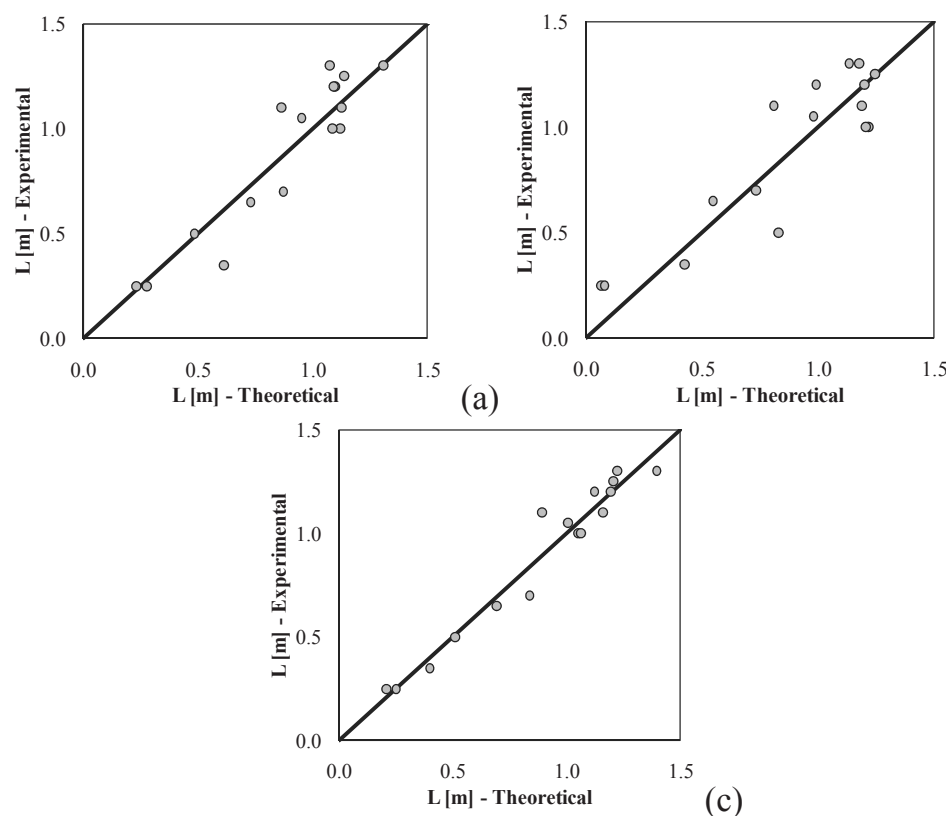

(b)

(c)

Figure 6: (a) Model 1 (eq. (8) and eq. (14)); (b) Model 2a (eq. (19)); (c) Model 2b (eq. (20)). 
in a stepped chute inclined in $45^{\circ}$. The equations were adjusted using a set of data obtained for 16 different experimental conditions, in which the flow rate and the opening of the sluice gate were changed. Predicted and measured values showed adequate superposition for the three models. The best superposition was obtained for the second modified model.

\section{Acknowledgements}

The authors are indebted to FAPESP (Fundação de Amparo à Pesquisa do Estado de São Paulo), CAPES (Coordenação de Aperfeiçoamento de Pessoal de Nível Superior) and CNPq (Conselho Nacional de Pesquisa e Desenvolvimento Científico e Tecnológico), for financial support of this study.

\section{References}

[1] Boes, R.M. (2000). Two phase flows and energy conversion in large stepped channels. PhD Thesis. ETH Zurich, Zürich Switzerland [in German].

[2] Boes, R.M., Hager, W.H. (2003). Two-phase flow characteristics of stepped spillways. J. Hydraulic Eng. 129(9), pp. 661-670.

[3] Schulz, H.E.; Simões, A.L.A. (2011) "Alterative equations for transition lengths in aerated flows: analogies with basic equations of transport Phenomena", Lab. of Reology and Turbulence, School of Engg. at São Carlos, Univ. of São Paulo, LTR Report I/I/11, (vertedoresemdegraus. blogspot.com;stoa.usp.br/ltr/files/) [in Portuguese].

[4] Simões, A.L.A.; Schulz, H.E.; Porto, R.M. (2010) "Stepped and smooth spillways: resistance effects on stilling basin lengths" Journal of Hydraulic Research 48(3), pp.329-337.

[5] Cain, P.; Wood, I.R. (1981) Instrumentation for aerated flow on spillways. ASCE, J. Hydraulic Eng. 107, No HY11. 